UNIVERSITE DU QUEBEC A CHICOUTIMI

\author{
COMMUNICATION ACCOMPAGNANT L'DEUVRE \\ PRESENTEE A \\ L'UNIVERSITE DU QUEBEC A CHICOUTIMI \\ COMME EXIGENCE PARTIELLE \\ OE LA MAITRISE EN ARTS PLASTIQUES
}

PAR

EDITH BERGERON

\title{
HOMME DE PAPIER
}

juin 1992 


\section{Bibliothèque}

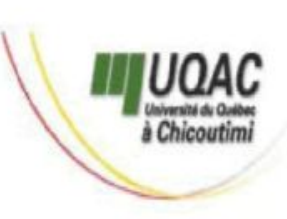

\section{Mise en garde/Advice}

Afin de rendre accessible au plus Motivated by a desire to make the grand nombre le résultat des results of its graduate students' travaux de recherche menés par ses research accessible to all, and in étudiants gradués et dans l'esprit des accordance with the rules règles qui régissent le dépôt et la governing the acceptation and diffusion des mémoires et thèses diffusion of dissertations and produits dans cette Institution, theses in this Institution, the I'Université du Québec à Université du Québec à Chicoutimi (UQAC) est fière de Chicoutimi (UQAC) is proud to rendre accessible une version make a complete version of this complète et gratuite de cette œuvre. work available at no cost to the reader.

L'auteur conserve néanmoins la The author retains ownership of the propriété du droit d'auteur qui copyright of this dissertation or protège ce mémoire ou cette thèse. thesis. Neither the dissertation or Ni le mémoire ou la thèse ni des thesis, nor substantial extracts from extraits substantiels de ceux-ci ne it, may be printed or otherwise peuvent être imprimés ou autrement reproduced without the author's reproduits sans son autorisation. permission. 
Ce travail de recherche a été réalisé

à l'Université du Québec à Chicoutimi

dans le cadre du programme

de maitrise en arts plastiques extensionné

de l'Université du Québec à Montréal

à l'Université du Québec à Chicoutimi 


\section{Résumé de lo communication accompagnant loeuyre d'Edith Bergeron présentée dans le cadre de la Maitrise en Arts Plastiques}

"Homme de Papier": Quinze sculptures de papier, dont cing matricielles faites de carton recyclé, sont l'objet de mon exposition. Quinze cônes: cônes phalliques, éros générsteur multipliè et répété. "Homme de Papier", cosmos vivant en perpétuelle regénération. Symbole vivant et sacré du caractère cyclique et dynamique de l'evolution cosmique.

11 faut voir ma protique srtistique comme une démarche s'inscrivant dans une recherche globale. L'exposition de ces sculptures, Homme de Papier) c'est d'abord le résultat d'une recherche plastique suivie d'une communication écrite tentant d'établir un parallèle entre l'Homme et son univers. De ce fait, il m'apparait avoir, à la lumière de ces oeuvres, une plus grand compréhension de l'humain.

Depuis près de 1900 ans, on s toujours connu le papier comme le support privilégié de l'écriture et de l'oeuvre picturale. Mais depuis environ vingt ans, le papier est lui-même devenu un médium, un matériau plastique. D'abord support des idées et du langoge, il devient pour l'artiste cantemporain sujet et objet de creation; propriétaire autonome de son discours.

Aujourdhui, la fabrication artisanale du papier se veut un hommage à tous ses artisans, grâce à qui la somme du savoir fut colligée et sauvegardée par ces espèces d'extensions du cerveau humain. Grâce au papier, à l'écriture, à l'impression et aux liures: la connaissance, le savoir et la culture, richesses incommensurables accumulées par l'humanité toute entière, font désormais partie du patrimoine universel. Le papier a toujours joú un rôle moteur et capital dans l'évolution de l'humanité, dans tous nos dispositifs de savoir et de pouvoir.

L'histoire de l'univers, de la terre, de l'homme, c'est aussi l'histaire de "Homme de Papier". L'histoire du monde, de 
I'homme a un sens. Teilhard de Chardin dans sa théorie de l'évolution le demontre clairement. Ce grand scientifique, paléontologue et philosophe, se pose la question: quelle est la place de l'homme sur la terre, quel est son rôle et quel est son destin?

11 y eut d'abord un progrès dans l'organisation de l'inanime, des atomes aux molécules plus complexes, (pré-vie) dont le sens était de préparer la vie. Puis, tout a changé d'ordre brusquement: le "pas de la vie" fut franchi. Imperceptiblement la vie a évolué; la cellule, comme l'atome se sont préparés a leur tour. Des organismes plus complexes et mieux doués "psychiquement" sont apparus: ainsi se preparait l'Homme. L"Homme dont la nature est le couronnement de cette même nature dont il est solidaire, qu'il domine et organise en lui donnant un sens. "Homme de Papier", à la fois fragile et puissant, en perpétuelle évolution. Artisan de sa propre ascension psychique ou de sa déchéance progressive. Chaque atome si petit soit-il, rayonne au moins la valeur de son volume. Si chacun des atomes, les uns près des sutres, rayonnent chacun leur volume, il y a nécessairement interaction. Dans mes sculptures, ces arbres de vie, ces cônes du temps, le seul moyen pour un élément de se rapprocher des éléments voisins est de resserrer le cône et de faire mouvoir vers le sommet ce fourmillement individuel. Mais, il y aura toujours ces abimes se creusant entre les cimes, ces inévitables souffrances, cette possibilité apocalyptique pour l'humanité de s'annihiler. Cependant, comme l'infiniment petit, la ploretorisation ne peut qu'avancer un peu plus dans le sens d'une unanimité grandissante. Plus complexe, l'humanité entière émerge au coeur d'un système toujours plus vaste d'éléments plus nombreux et mieux orgonisés. Elle cherche le sommet du cồne aspirée par une force, par un Absolu qui la transcende.

"Homme de Papier" temoigne de la nécessaire résurgence cyclique. L'arbre est le lien indispensable entre la terre où il plonge ses racines et l'air qu'il touche de so cime. La survie de l'homme est indissociablement liée à la survie de la forêt. Quinze sculptures morphologiquement humanoïdes, arborescentes, ovoïdes; à la fois uterines et phalloides a base circulaire. Cing objets matriciels faits de milliers de petits cartons recyclés qui ont engendré des sculptures faites de papier-matière (de l'abaca) teint intégralement 
de noir (5) et couleur chair (5). Cône matrice, creux utérin, cavité où la vie prend forme, où se développe le foetus. Cône phallique, éros générateur, géniteur, qui répète. Cône thanatos, ogival, cône mamie, sclèrose de la vie. Cône volcan du magma contenu, là où les pulsions incandescentes surgissent des entrailles de l'inconscient. Cône recueillement, gravité bénédictine de l'orant. Permanence, pérennité du menhir. Supplique, ronde incantatoire du derviche tourneur. "Homme de papier", cosmos vivant en perpétuelle regénération. Cartons recyclés, imbriqués les uns aux autres. Geste mille fois répété pour marquer un rythme, une suite, un aboutissement. Patience dans le temps, dans la gestation du monde, dans la continuité de l'univers. Recreer, tisser sa structure, son mouvement.

Papier-matière: molécule, fibre et verbe. Papier-mémoire de l'humanite, papier témoin de lhomme, moteur de sa fulgurante évolution, intellectuelle et psychique. Artiste/Dieu qui dans l'agitation créatrice voit surgir du liman de fibres indifferenciées une image miroir, une direction, une création. A partir de l'organique, la matière prend sens, s'organise, parle. Le papier-matière en formation devient un centre atomique moléculaire de l'univers. Comme celui-ci, le papier-matière est traversé d'un courant qui le contraint à produire de l'ordre, à organiser le cosmos. Le papier est mü par une energie lui permettant dybluer yers alus de conscience 11 apporait vibrant, chaud, potelé, ridé, docile, disponible, doué de virtualités insoupçonnées.

Mes sculptures de papier/chair, évoquent la chaleur originelle qui a permis l'incubation cosmique de sortir du chaos initial. Agent de renaissance, de vie et de communication. Energie qui fait mürir biologiquement et spirituellement.

Papier/noir, noirceur des ténèbres abyssales des eaux profondes qui précèdent la création, réservoir de toutes choses, mais surtout de la vie latente. Ventre de la terre, matrice du "si le grain ne meurt". Blessure, ouverture pour atteindre le caché, l'anima, l'inconscient, la source, la nuit qui inspire. Connaître la nuit et revenir à la purification spirituelle où l'inconscient se libère, où la germination, riche de toutes les virtualités de l'existence éclate en manifestation de vie. Papier fragile, papier tressaillement porteur d'espoir 
renouvelé. Floraisons spiralées qui sortent du point originel et qui se prolongent à l'infini, reliant ainsi les deux extrémités du devenir.

Le concept "Homme de Papier" dévoile la nécessaire obligation pour tous les etres pensonts de travailler de toutes leurs forces pour atteindre individuellement et collectivement le sommet du cône. "Homme de Papier" suit l'Homme dans sa marche qui l'élève de son essence brute et élémentaire jusqu'à cette montée transcendante. Le chemin est-il difficile, l'issue est-elle incertaine, est-il question de progressions lucides ou de déchéances dans l'obscurité?

"Homme de Papier", complexe comme l'univers, comme la vie. complexe comme l'Homme qui approche du mystère ou de la révélation. comme l'artiste, il capte et transmue la substance pour la projeter dons l'infini de l'espoce. 
TABLE DES MATIERES

1. Introduction 1

11. Chapitre 1

Le papier 5

111. Chapitre 11

L'homme

12

14. Chapitre 111

Homme de papier

22

y. Conclusion

32

४1. Bibliographie

34

V11. Annexe 


\section{INTRODUCTION}

Ma véritable découverte du papier comme médium artistique date de 1984. J'ai bequcoup aimé et apprécié cette exposition et toutes les possibilités de création artistique de ce médium. Après la pierre, le bois, le métal, voici le papier-matière; matériau vivant à la fois fort et fragile.

La fabrication artisanale du papier fait partie intégrante de mon aeuvre. A toutes les étapes de lo création, je mappliqueroi à extraire du papier tout son potentiel, toute la richesse physique de sa structure, en un mot, toute sa quintessence. Fibres d'abaca déchiquetées, réduites en fines particules, broyées, mélangées avec de l'eau. Cette pâte est répandue d'abord sur un treillis métallique où elle s'égoutte lentement, ensuite elle est déposée sur une table drapée qui permet un séchage efficace.

Lentement les fibres se tassent, les molécules se regroupent, la matière prend vie, s'organise, prend forme. Le papier-matière apparaît lisse ou rugueux, gaufré, chaud et sensuel au toucher, tout 
prêt à recevoir le souffle de l'artiste.

La conscience aiguë de l'apport inestimable du papier dans notre vie m'a incitè à utiliser du papier recyclé (boites de carton) pour produire les cing sculptures initiales qui servent de matrices aux deux autres series de sculptures. Récuperation, survie de ces milliers de morceaux de carton dont la valeur symbolique nous donne à comprendre qu'une fois réunis, ils sont appelés à former un système plus complexe comme le cône de l'humanité qui se prolonge au-delà de nos individus.

Par la sociologie, la philosophie, la métaphysique, je suis à la recherche d'une plus grande compréhension de l'Homme et de son univers. Ma demarche artistique s'inscrit dans cette direction. "Homme de Papier" est la résultante de cette interpellation personnelle. Je veux observer l'Etre humain dans son univers, son environnement, établir ses rapports avec son milieu, faire ressortir ses sentiments, son intériorité, signifier la grandeur et la misère de l'Homme. "Homme de Papier" à la fois fragile et puissant, en perpétuelle évalution, artisan de sa propre ascension psychique ou de sa déchéance progressive.

Dans la première partie de mon travail, je fais un bref historique du papier jusqu'ò l'avènement de l'imprimerie de Johannes Gutenberg et du livre. C'est un hommage au papier, à l'impression et au volume grâce à qui la connaissance, le savoir et la culture, richesse 
accumulée par l'humanité toute entière font désormais partie de notre potrimoine universel.

Le discours philosophique de la deuxième partie: l'Homme, se veut une réponse à cette préoccupation existentielle, d'une meilleure compréhension de l'Homme et de son univers. La pensée de Teilhard de Chardin vient appuyer et soutenir le propos.

Enfin la troisième partie Homme de Papier est la somme des deux premières parties, la résultante. Je fais d'abord ressortir un parallèle entre l'Homme et "Homme de Papier". Par la suite je fais appel à la sémiologie pour démêler "l'écheveau d'abaca" et expliquer la crégtion d'une nouvelle trame (papier-matière) qui donnera naissance à "Homme de Papier". Je suggère en tentant, autant que faire se peut, d'eviter tout discours exhaustif, toute grille, code ou certitude mathématique. 
LE PAPIER 


\section{Chapitre 1}

\section{LE PAPIER}

Les matériaux successivement utilisés par l'homme comme support de sa pensée furent la pierre, la tablette d'argile, la plaquette de bois, le rouleau de papyrus utilisé en Egypte dès l'an 3000 avant J.-C., la feuille de parchemin à Pergame vers 300 avant $J .-C$, enfin, le vélin (peau de mouton ou de veau). Parmi les autres supports destinés à recevoir l'écriture, on peut aussi inclure le bambou qui était considéré par les Chinois, trop lourd et la soie, trop onéreuse. La tradition place, vers l'an 105 après $J .-C$., l'invention du papier en Chine par Ts'ai Lun, fonctionnaire de l'empereur Ho-Ti. 11 réalise la première pâte à papier à partir de tissus rebutés, de lin et de chanure, de chiffons, de l'écorce de mürier ou de toile à voile et des filets de pêche usagés. La pâte obtenue par macération et foulage à la main et à la massue était étalée en couche mince sur un tamis fibreux où elle s'égouttait et séchait.

Le papier est né mais le secret de sa fabrication sera jalousement gardé en Extrême-0rient pendant près de 600 ans. La 
diffusion et l'utilisation du papier, s'accomplit en Chine dès les premiers siècles de notre ère. Elle apparaitra au Japon au début du Vle siècle sous le règne de l'imperatrice Suiko (593-628). Elle gagne ensuite et toujours plus loin vers l'ouest au hasard des échanges commerciaux ou des expéditions militaires qui forçaient les frontières des peuples voisins. Les Arabes font prisonniers aे Samarkand des soldats chinois papetiers de métier, qui pratiquent leur sovoir faire dans la cité. C'est ainsi que successivement on retrouve le papier à Bagdad, Damas et au Caire où, vers 900, l'usage du papyrus est presque complètement disparu. Suite aux conquêtes arabes, bientôt, se propagera en Afrique du Nord, en Sicile et en Espagne, la fabrication du papier. Ce sera à Fès au Maroc en 1100 et à Tartiva, aujourd'hui San-filipé en Espagne en 1150. On dit que cette date marque probablement l'introduction du papier en Europe. Par la suite, des croisés français prisonniers des Arabes, sont contraints de travailler dans les papeteries de Damas. Lorsqu'ils furent libérés, ils apportèrent en France le fruit de leurs expériences. Vers 1157, à Vidalon et en 1189 à Lodève dans le département de l'Hérault. Ces deux dates sont plus ou moins cautionnées par les historiens. Plus tard, le papier sera fabriqué à Fabriano (Italie) en 1276: "ce sera le premier moulin à papier européen". (1)

Les filigranes, dessins visibles dans la feuille par transparence, apparaissent vers la fin du Xllle siècle. L'histoire du papier s'écrit

1 Hunter, Dard, Papermaking, Dover Publication, p. 474 
alors avec beaucoup plus de certitude; le filigrane permet d'en établir la provenance, le format, l'époque et la période approximative de fabricstion. D'autres moulins s'implanteront en France vers 1348. Mentionnons Soint-Julien près de Troyes et Essonnes. L'Allemagne suivra avec un premier moulin à papier a Nuremberg en 1390 et. l'Angleterre en 1495. L'invention de l'imprimerie par l'Allemand Johannes Gutenberg vers 1450 ya augmenter considerablement la demande du papier a cause de la très grande diffusion de la bible, maintenant devenue possible.

Par la suite, le papier sera soumis a la machine (invention de la machine a papier en 1798) par le français Nicholas-Louis Robert et va devenir un besoin industriel, une chose, un produit de consommation.

"C'est la réification totale; son usage ne sera plus magique ou ludique, son espace sera celui de quelques "formes" normalisees, voire une simple largeur. fabriqué en rouleau pour l'impression "en continu", son poids évalué en grammes / millimetres, son corps, ses couleurs figées, ses usages démultipliés jusqu'à des fins dites "hygiéniques". (2)

2 Gheerbrant, Bernard, Trayerses, 127-28, p. 60 
Ce n'est pas ce papier industriel "dénaturê" qui m'intéresse. C'est d'abord la pâte signifiante, le papier-matière, fait main, de fabrication la plus artisanale qui soit (yoir annexes $1,2,3 \ldots$ ) Matériau de base, limon de la création prêt à déliurer une forme, un symbole, un sens. D'abord support de l'écriture et de l'impression, il devient pour l'artiste contemporain objet et sujet de creation.

Depuis le début des années 70 , de plus en plus d'artistes utilisent le papier comme matériau privilégié à la création artistique. lls le sortent de sa gangue industrielle, certains le recyclent et le recupèrent. Le papier tout comme la pierre, peut signifier par lui-mème. Avec "Homme de Papier", j'ai tenu à émanciper la matière-papier, à l'aimer "pour elle-même". J'ai voulu libérer sa plus grande énergie. L'exulter en canalisant ce potentiel caché de la matière, cette force enfouie du cosmos.

\section{Papier fait main}

J'utilise la fibre d'abaca comme matière première, comme matériau de base. Le rapport entre les fibres et l'eau est d'environ $0,5 \mathrm{~kg}$ de fibres pour 100 litres deau. Lẹ fibres sont déposées dans une cuve au $2 / 3$ remplie d'eau. Elles sont par la suite hachées, déchiquetées, brisées, triturées par le malaxeur jusqu'au défibrage complet. A la pâte obtenue, s'ajoute un agent de rétention et lo teinture.

La pâte de couleur en suspension apparaît, homogène, informe, ò 
la limite de la saturation. Cette pâte saturée d'eau, est déposée sur un tamis métallique qui retient une couche de fibres emmêlées permettant ainsi la première et la plus grande partie de l'égouttage. Le contenu du tamis est "renversé" sur une table préalablement drapée. L'opération est plusieurs fois répétée. Je travaille ensuite ayec une éponge, ce "froid magma" qui petit a petit va prendre forme, prendre vie. La table legèrement inclinée et une ventilation adéquate permettent un dernier égouttage un séchage complet. Habituellement, après vingt-quatre heures, la feuille de papier est prête à recevoir le souffle de l'artiste. Encore toute chaude, fébrile, couverte de granulations à palper, marquée par l'écriture du hasard, de signes à décrypter, de lectures virtuelles. La feuille s'offre aux sens. La fabrication artisanale du papier est un hommage rendu à tous les artisans du papier fait main qui remante jusqu'à Ts'ai Lun. Depuis des centaines d'annees, le papier a joué un rôle moteur, capital dans l'evolution humaine, dons tous nos dispositifs de savoir et de pouvoir. De ceux-ci, il en est devenu même le symbole. L'âge du papier commence veritablement après la découverte de l'imprimerie par Johannes Gutenberg. Ce sera l'irréversible prolifération des savoirs et de la connaissance: la culture, la civilisation par l'écrit. Tout au cours des siècles, le progrès humain sera indissociable du livre, véritable support de l'information et de la communication. Les livres s'engendrent les uns les autres, le savoir s'interpénètre dans toutes les civilisations. Hommage à cette "mémoire" de l'Homme, à cette noble "extension" de son cerveau qui a permis une telle accumulation de connaissances. Hommage aussi à l'Homme, fruit et aboutissement 
de la longue gestation de la terre qui se prend en charge et devient l'artisan de sa propre destinée. 
L'HOMME 


\title{
Chapitre 11
}

\section{L'HOMME}

\begin{abstract}
"Comment ne pas voir que, après nous avoir roulés individuellement chacun de nous-vous et moi sur nous-mêmes, c'est toujours le même cyclone (mais ò l'échelle sociale, cette fois) qui continue sa marche au-dessus de nos têtes-nous resserrons tous ensemble dans une étreinte qui tend à nous parfaire chacun, en nous liant organiquement à tous les autres à la fois. (3)
\end{abstract}

Molécule, granulation, particules en suspension qui s'agglutinent, s'entremêlent, s'accrochent à une structure. Une mystérieuse identité les englobe et les cimente. Aucune fibre élémentaire n'est entièrement indépendante dans sa croissance de fibres voisines. Chacune d'elles apparait prise dans une faisceau plus grand encore. Le désordre apparent se polarise, s'organise, cherche so synthèse, son sommet, celui du cöne, aspire en quelque sorte par une force qui la transcende.

3 Teilhard de Chardin, le Phénomène humain, p. 339 


\begin{abstract}
"Aux corpuscules cosmiques nous trouverions naturel d'attribuer un rayon daction individuelle aussi limité que leurs dimensions mèmes. or il devient evident ou contraire que chacun d'eux n'est definissable qu'en fonction de son influence sur tout ce qui est autour de lui. Quel que soit l'espace dans lequel nous le supposions placé, choque élément cosmique remplit entierement de son rayonnement ce volume lui-même.Si étroitement circonscrit donc que soit les "coeur" d'un atome, son domaine est coextensif, ou moins virtuellement a celui de n'importe quel autre atome. Etrange propriété que nous retrouverons plus loin jusqu'à la molecule humaine" (4)
\end{abstract}

Les atomes ne sont pos indépendants entre eux; quelque chose les relie les uns aux autres qui les fait solidaires. Sans être encore tout à fait de la matière, une mystérieuse identité les réunit. Ce dedans des chases apparait avec évidence chez l'Hamme : c'est la conscience, le psychisme. Mais "la matière originelle" est aussi quelque chose de plus que le "grouillement particulaire".

"Au fond de nous-mêmes, sans discussion possible, un interieur apparait par une déchirure, su coeur des êtres. C'en est assez pour qu'à un degré ou à un autre cet "intérieur" s'impose comme existant partout et

4 Teithard de Chardin, le Phénomène humain, p. 35, 36 
depuis toujours dans la nature. Puisque, en un point d'elle-même, l"Etoffe de l'Univers a une force interne, c'est forcement qu'elle est biface par structure, cest-à-dire en toute region de l'espace et du temps, aussi bien par exemple que granulaire: Coextensif à leur Dehors il y a un Dedans des choses". (5)

Dans l'histoire de la terre, il y a eu d'abord un progrès actif et rayonnant de l'organisation de l'inanimé, des atomes aux molécules plus complexes (pré-vie) dont le sens était de preparer la vie. Puis, tout a change d'ordre brusquement: le "pas de la vie" fut franchi. imperceptiblement la vie a évolué; comme l'atome de la matière inorganique, la cellule s'est complexifiée à son tour. Des organismes de mieux en mieux organisés sont apparus; de l'unicellulaire au primate; toute une serie d'êtres intermediaires dont le dedans (psychisme) devait se complexifier au même rythme que le dehors: ainsi se préparait l'Homme. L"Homme dont la nature est le couronnement d'une nature dont il est solidaire et qu'il domine et organise en lui donnant son sens. Mais souvent, il ira même jusqu'à dénaturer la nature, au détriment de tout autre vie: tant végétale qu'animale et tarir ce qui est à la source même de notre survie: l'eau.

5 Teilhard de Chardin, le Phénomène humain, $0.52,53$ 
Le pas de la vie, le pas de la réflexion sont des sauts de complexité interne, ou l'intérieur plus complexe devient capable de plus d'intériorité. "Préconscience" "bioconscience", conscience réfléchie. L'Homme n'est superieur aux autres ètres de la nature que par sa psyché. Par une sorte de loi de "complexité-conscience", le progres de conscience est proportionnel à l'organisation et en particulier à la complexité cérébrale.

Contrairement aux animaux, l'Homme sait qu'il sait. De cette faculte nouvelle, émerge tout un réseau d'aptitudes et de propriétés nouvelles: liberté, prévision de l'avenir, aptitude à construire, à créer. I1 y a une analogie, une certaine parenté entre le cerveau humain formé de milliards de cellules enchevêtrées et "lappareil pensant social" ayec ses milliards de cellules réfléchissant solidairement. Sur les cinq continents, la Connaissance accumulée au cours des siècles s'échange, dans un extraordinaire réseau de communications écrites (livres) et médiatiques (audio-visuelles). Seul, IHomme ne pourrait imaginer, circonscrire et maitriser cette somme de connaissances; mais, par ailleurs, il pose des gestes individuels, participe pour lui-même et pour tous au développement et à la montée générale de la conscience. La pulsion de cette évolution de conscience universelle est alimentée par une énergie inscrite dans nos gênes. Pierre Teilhard de Chardin la nomme: "l'amour-énergie". L'évolution est "amorisante". Comme un verticille, comme l'épiderme du papier où chaque partie si distincte soit-elle demeure jointe aux autres dans une trame commune l'Homme est à la fois unique et 
solidaire. Atomes, molécules, "inter-fécondation" à tous les degrés, brassage de gènes, de races, de civilisation, l'Homme a réussi à faire ce qu'aucune autre espèce syait fait avont lui: "couvrir, sans se rompre, la Terre d'une seule membrane organisée". (6)

Lentement, par le jeu de forces irrésistibles, les "feuillets humains" continuent à se refermer, ì s'enrouler autour de nous. Malgré certains ratés de la pulsion primale (Guerre at sawhoncel le cône de l'Humanité sorgonise siblye chaque molécule humaine solidairement tend vers un absolu, une force qui transcende.

"Incontestablement, à une vitesse
toujours accélérée, le réseau cun
reseau mondial) des liens
économiques et psychiques se tisse,
et nous enserre et nous pénètre, plus
etroitement sans cesse. Chaque jour
un peu plus, il nous devient
impossible d'agir et de penser
autrement que sous une forme
solidaire. Que signifie cette
étreinte multiforme, à la fois
externe et interne, contre laquelle
nous nous débattons en vain?
Serait-ce par hasardque, pris dans
un engrenage aveugle, nous soyons
destinés à périr étouffés sur
nous-mêmes? - Non. Car à mesure

(6) Teithard de Chardin, le Phénomène humain 
que l'enroulement se resserre et que la tension monte, une échappée utile se découvre, dans l'immense genérateur aux forces de sur-compression". (7)

Est-il possible d'atteindre une certaine solidarité humaine si chaque individu, chaque saciété he voit dans son vaisin qu'une "parcelle close"? Finalement ces attitudes, bien qu'individuellement raisonnable, ne serait-elle pas inscrite dans une trajectoire qui mènerait éventuellement au désordre, aux conflits, à la guerre et au chaos total? Pas nécessairement, nous accédons à la cime en nous serrant les uns contre les autres, en nous retrouvant et en nous achevant nous-mêmes dans un autre que nous.

Dans cet artire de vie, ce cône du temps, le seul moyen pour un individu de se rapprocher et d'être solidaire avec les autres, est d'éviter l'éparpillement, resserrer les liens et pousser cullectivement ce faurmillement individuel vers le sommet du cône.

Mais il y aura toujours ces "abimes se creusant entre les cimes". Cette inévitable souffrance sous toutes ses formes: la pollution, la guerre, la falie nucléaire, la possibilité apocalyptique pour l'humanité de s'annihiler. C'est avec labeur, peine, que se rassemble, se dégage,

7 Teithard de Chardin, le Phénomène humain, 218 
s'épure la terre nouvelle. Ce "mal de croissance" se retrouve à tous les échelons de l'evolution de l'Homme et de l'univers. Les affres de "l'enfantement" se retrouvent à tous les echelons de l'échelle de l'évolution; a partir des simples molécules jusqu'aux plus hautes syntheses de l'esprit. Ce mal nécessaire qui fait se traduire en termes de travail et d'effart tout progres.

"Univers qui s'intériarise mais aussi
du même mouvement, Univers qui
peine, Univers qui pèche, univers qui
souffre... Arrangement et centration:
double opération conjuguée qui,
pareille à l'ascension d'un pic ou à la
conquête de l'air ne peut
objectivement s'effectuer que si
elle est rigoureusement payee, -
pour des raisons et suivant un taux
tel que, si nous pouvions les
connaitre, nous aurions penétré le
secret du Monde autour de nous". (8)

Pour la réussite de l'effort universel, il est inévitable qu'il y ait des individus douloureusement arrêtés dans leurs possibilités et leurs efforts. Il est inévitable dans chaque mentée, qu'il y ait de la peine, du mal, de la souffrance, beaucoup d'insuccès, beaucoup de blessures. La souffrance serait-elle en définitive la force ascensionnelle du monde caché? Serait-elle la forme la plus haute que pouvait prendre

8 Teilhard de Chardin, le Phénomène humain, p. 347 
l'oeuure mystérieuse de la création?

L'Homme correspond bien à cette "étoffe cosmique" portée à un état superieur et toujours croissant de complexite et de conscience. Même si la masse humaine semble aujourd'hui dispersée et que les élements de cette fourmilière agitée semblent se repousser les uns les autres, il ne faut pas desespérer. La terre entière est un grand corps en train de naitre dans la douleur qui ne va pas en se dissipant mais en se concentrant sur elle-même comme toutes les formes vivantes que nous connaissons. La conscience planetaire ne peut qu'avancer toujours un peu plus dans le sens d'une "unanimité grandissante". L'Humanité tisse son cerveau; elle émerge au coeur d'un système toujours plus vaste, d'éléments plus nombreux et mieux organisés. Elle cherche le sommet du cône, aspirée par une force, par un Absolu qui lo transcende. 
“... L'Humanite prise dans son ensemble deura, comme il était arrive sux forces individuelles de l'instinct, se réflechir à son tour "ponctuellement" sur elle-même (c'est-à-dire dans ce cas abandonner son support organo-planetaire pour s'excentrer sur le centre transcendant de sa concentration grandissente), alors, pour l'Esprit de la Terre, ce sera la fin et le couronnement". (9)

"Homme de Papier", qui est aussi le titre de mon oeuvre se veut, tout bien considéré, le reflet, le miroir, le signe iconique de l'Homme. 
HOMME DE PAPIER 


\section{Chopitre 111}

\section{HOMME DE PAPIER}

"Dans un même mirair ou une même pupille, il y a limage de tous les abjets placés devant et chacun de ces objets est tout en toute la surface du miroir et tout en chacune de ses moindres parties. En tous lieux ou le soleil voit l'eau, l'eau voit également le soleil, et en chacune de ses parties elle peut présenter à l'oeil l'image du soleil"

Les principaux éléments de la communicstion humaine, sont la parole et l'ecriture. Mais lorsque la communication prend la forme de signes iconiques elle devient une des nobles facettes par où s'exprime l'être humain. Cependant, il faut être prudent et modeste quand on propose une lecture d'un message sculptural, un code d'analyse sémiologique.

L'art est le refuge de la sensibilité, de la spontanéité et du plaisir de créer. Décoder une oeuure d'art par des justifications 
trop discursives risque d'occulter plutôt que de mettre en lumière lintention premiere. 11 est periols difficlle pour lartiste de restituer ce monde cénesthésique de tensions, d'émotions et de projet qui aboutit a loeuvre. Pendent tout le processus de crétion, l'artiste nessele pas de déceler ses raisons profondes, de cerner tout ce qui s'agite en lui, tout ce qu'il sent, pense et souhaite. Ce serait la mellleure facon de freiner son élen et de le neutraliser. Après coup. l'artiste peut toujours, a lo maniere dun critique, descendre à la source et tenter d'analyser avec lucidite la seve qui le nourrit.

\begin{abstract}
"Un arbre ne sait pas qu'il est un arbre; il elabore sa matière et sa forme; il etire ses branches a 10 conquête de l'espace; il épanche son feuillage pour pousser sa sevevers l'air qu'il respirera. II se borne è etre le champ actif de cet enorme echange et d'en tirer sa splendeur et so force. Cela dispense-t-il le botaniste de l'étudier dans son espèce, dans son fonctionnement, dans sa logique vitale?" (11)
\end{abstract}

L'artiste peut tenter d'expliquer avec des mots ce qui s'agite au plus profond de lui-même, mais ce ne sera quiune vérité. En expliquant tout de l'oeuvre, celle-ci nest plus ouverte sur l'inconnu.

11 Hugghe, René, les puissances de limage, p. 119 
J'éviterai done toute lecture systemetique, toute anelyse discursive de "Homme de Papier". Je disposerai plutot de balises indicielles ou toutes les avenues, chemins, traverses seront permis. "Homme de Papier" n'existera que dans le réclt que j'en donnerai et dens la somme de toutes les lectures quion pourra y faire.

Si l'expression plastique est un langege, il n'est pas aisé dy établir sa grammaire, son lexique, sa sémantique; de mettre dun côté les signifients de l'oeuvre, de l'autre ses signifiés. La création artistique ne peut etre réduite dun systeme. Je n'essaierai pas de décrypter; de chercher la clef, de concevoir la description de "Homme de Papier" comme un état neutre, litteral, mais bien d'en faire un lieu d'investissements subjectifs. "Homme de Papier" n'est ni un objet réel, ni un objet imaginaire. L'identité de ce que je veux représenter est sans cesse répétée, renvoyée. Le signifié est donc toujours déplacé.

\footnotetext{
"Avec Schefer qui prolonge sur ce point fondamental le travail de Julia Kristeva, La sémiologie sort encore un peu plus de l'ère du Modèle, de la Norme, du Code, de lo loi - ou silion prefère: de la théologie". (12)
}

12 Barthes, Roland, Lobvie et lobtus, p. 140 
"Homme de Papier" a sa propre théorie; il n'est pas lexpression d'un code ni un dépôt d'un système mais générateur de systèmes. L'infinité de lecture quon peut y faire, lui donne une infinité de langage; cette enalyse sans fin est précisement son système.

Quinze sculptures morphologiquement humanoides, arborescentes, ovoildes; à la fois utérines et phalloides à base circulaire. Cina matricielles faites de milliers de petits cartons recuclés qui ont engendré dix autres sculptures faites de pepier-matière (abaca) teint intégralement de noir (5) ou couleur chair (5).

Trois et cing, chiffres universels et fondamentaux. Freud voit un symbole sexuel dans le nombre trois. Triade dans lequelle apparaissent les rôles de Père de Mère et d'Enfant. La création artistique implique aussi le créateur, l'scte de créer, la créature ou loeuure.

$\begin{array}{lll}\text { Ciel } & \text { Terre } & \text { Homme } \\ \text { Père } & \text { Mère } & \text { Enfant } \\ \text { Prévie } & \text { Vie } & \text { Survie } \\ \text { Esprit } & \text { Corps } & \text { Ame } \\ \text { Naissence } & \text { Croissence } & \text { Mort } \\ \text { Apperition } & \text { Evolution } & \text { Destruction } \\ \text { Inconscient } & \text { Conscient } & \text { Supraconscient } \\ \text { Matériel } & \text { Rationnel } & \text { Spirituel }\end{array}$


Chiffre impeir qui n'exprime pas un état mais un acte. Le quinaire est le nombre de la création et de l'individualité. Le cinq est aussi le symbole de lhumsin au terme de son evolution biologique et spirituelle.

Lo symbolique du pentegramme est multiple et elle a toujours comme base le chiffe cinq qui exprime lunion des inégeux: "Les cinq branches du pentagramme, accordent en une réunion féconde le 3 , qui signifie le principe mâle et le 2, qui correspond au principe féminin. Il symbolise alors l'androgynie.

Cône symbole, cône cocon, larve de forme "présignificative", "Parabole conique", pré-balbutiement vital. Cone de "l"arbre de vie". Symbiose du substrat mâle et femelle. Cône matrice, réceptacle, creux utérin où la vie prend forme, ou se developpe le foetus. Cavité qui contient, preserve, protège; nid, giron maternel qui reçoit et qui donne.

Cône phallique, éros générateur multiplié et répété Cône thanatos, ogival, cône sarcophage. Cône momie, sclérose de la vie, mort, attrait, ospiration à la sur-vie. Cône volcan du magma contenu; des pulsions incendescentes surgissant des entrailles de linconscient. Cone qui ploie mais se stabilise, qui continue sa montée. Recueillement, gravité bénédictine de l'orant. Permanence, perennité, témoignage du menhir. Supplique, ronde incantatoire du derviche tourneur. Centre, mouvement circulaire qui évoquele 
définitif, limmuable, la soif de la perfection, de l'absolu, de linteriorité et de l'éternité. Cercle humain, sphéricité de la terre en giration ou centre de lunivers. Homme, eboutissement et centre de l'évolution. Equilibre centré, intégré, ou ce qui differencie ne nuit pes 8 lunité Dynamisme ascensionnel oủ les ténèbres abyssales aboutissent à le lucidité humaine.

Inconscient / conscient / "surconscient". Métalangage, expression privilegiee de l'artiste que je suis et qui peut suive, traduire et guider lHomme dans sa marche transcendante. Angoisse de l'échec ou de lo réussite de cette marche individuelle et collective.

"Homme de Papler", pilier, colonne cosmique, centre et principe d'unite. Lien entre le haut et le bas, lhumain et le divin, support de vie, support du monde. Alpho (cone) et omego (cercle); totalité de lo connaissance, totalité de l'être, totalité de lespace et du temps. Alpha "préconscience", oméga "surconscience" terme de lévolution, vers la "noosphère", la sphère de l'esprit vers iaquelle convergent toutes les consciences.

"Homme de Papier", cosmos vivant en regenération. Mort et renaissance de l'arbre en perpétuelle évolution, en ascension vers le ciel. Symbole vivant et sacré du caractère cyclique et dynamique de l'évolution cosmique. II réunit en lui tous les éléments: eau, terre, air et le feu jailli de son frottement. Image monoique perfaite; à la 
fols phallus et matrice. Image archetypole du père par son tronc dressé vers le ciel. Image aussi de la mère, de la fertilité, de la matrice ou s'opère la gestation des bourgeons, des fruits. Refuge des nids doiseaux.

J'ai d'abord concu les cina sculptures matricielles qui sont faites de milliers de petits cartons recyclés, interrelies, imbriqués les uns aux autres. J'ai répété inlessablement ces gestes, pour marquer un rythme, une reprise, une succession, une suite. Patience dans le temps, dans la gestation du monde, dans la continuité de lunvers. Duplication, répétition, leitmotiv. Recréer, tisser la structure et le mouvement de lunivers. Faire sortir de ma propre substance, tout comme le fait laraignè qui batit sa toile delle-même. Résurgence, papier phénix carton recyclé, signifiant perpetuellement engendré. Individualités semblables et différentes que je monte patiemment en organisme de dimension planétaire. Vannerie collective, réflexion que je trame dans un enroulement cosmique. Evolution individuelle et collective de la conscience des êtres. Le cône matrice des reletions et des constructions humaines prend forme, s'élève, se dresse. "Homme de Pepier", démiurge en quête d'absolu domine "l'arbre de vie" et peut lui donner une direction. Cinq continents a la fois semblables et differents unis dans une cause commune dens leur lente et longue progression.

Le genre humain est l'eboutissement et l'émsnation de la nature. C'est pourquoi l'Homme ne peut agir sans se préoccuper de tout ce qui 
lentoure, de tout ce qui contribue a le rendre tel quil est. Il foit pertie intégrante de la nature. Toute velleité, pour l'Homme de fonctionner sans tenir compte de son environnement est vouée irrémédiablement à l'échec. Pour moi le papier est une production, une création de l'Homme. La forêt, par contre, est a la base de tout. L'arbre est le lien indispensable entre la terre ou il plonge ses racines et l'air qu'il touche de sa cime. Sens lui, l'être humain ne pourrait surviure, subsister. La survie de lHomme est "indissociablement" liée a la survie de la foret. La disparition de celle-ci a des conséquences sur le plan planétaire comme l'érosion intempestive des sols, une diminution d'oxygenstion et une pollution toujours plus grande. Les grandes compagnies papetières (particulierement nombreuses dans notre region) sont les principales responsables de la déforestation et une autre cause importente de is pollution. Qu'il suffise de mentionner les solvants, les ligueurs noires dens nos cours deau, lanhydrite sulfureux qui tue lo vie do source même, lo bisulfite, la soude caustique, etc. L'élimination des arbres entraine celle de milliers d'espèces végétales et animales. Sans une gestion prudente de le forêt, l'espèce humaine pourra disparaitre elle aussi. Ainsi donc, tout le long travail de levelution, qui répond par des inventions biologiques d'une incroyable variété aux pressions de l'environnement sera anéanti. Les cinq matricielles de "Homme de Papier" temoignent de la nécessaire résurgence cyclique, témoignent aussi de la vie; la forêt s'élabore à mesure que les arbres se répètent, que la mosoïque s'organise. 


\section{Papier-matière}

Papier prémisse et issue, début et fin, molécule et verbe. Papier-mémoire de lihomme, papier-témoin et moteur de sa fulgurante montée sociale, intellectuelle et psychique. "Homme de Papier", condensé, microcosme et "homocosme". Artiste-Dieu qui dans lagitetion creatrice voit surgir, a partir du limon de flbres indifférencies une image, une direction. Plaisir de l'artiste de palper linterieur de son materiau, la substance première, la force elémentaire. Plaisir de prendre part au combat des élements, de participer a cette force qui dissout et qui lie. La main de l'artiste prend veritablement conscience du lent mais régulier progrès de cette matière virtuelle. A partir de "l'inorganique", la matière prend sens, s'orgenise, parle. L'eau, source de vie, précéde ma création. Elle contient l'infinité des possibles, tout le virtuel, le germe des germes. Quand elle se retire, elle devient tout à coup une mervellleuse source de fécondation de l'âme. Elle a ce powvoir de faire resurgir les énergies inconscientes, les motivations secrètes et inconnues. Quelque chose d'insaisissable relie les particules, les elements les uns aux autres qui les fait solidaires et en même temps, une mystérieuse identité les englobe et les amalgame. Le papier-matière en formation devient un centre infinitesimal de lunivers. Comme celui-ci, il est troversé par un courant qui le contraint à produire de lordre, de l'organisetion. Comme lui, 11 est mû par une énergie lui permettant dévoluer vers plus de conscience. Le papier-matière apparait vibrant, chaud, potelé, ridé, docile, disponible, doué de virtualités insoupconnées. Avide, il veut prendre lo parole et 
exprimer la verité de son être.

Popier-peou couleur de chair, "toctilite" charnelle, intimité, sensualité, chaleur épidermique. Chaleur originelie qui a permis de sortir du cheos initial; qui a permis lincubation cosmique. Agent de renaissance, de communication. Energie crétrice qui fait mürir biologiquement et spirituellement. Ardeur intérieure, flemboiement spirituel.

Fapier-noir, noirceur des ténères abyssales des eaux profondes qui précèdent la crégtion, réservair de toutes choses, de lo vie latente. Ventre de la terre, matrice de "si le grain ne meurt", réceptacle de la fécondité. Souffrance, douleur nécessaire de la gestation, de l'enfantement. Force essentielle à tous les niveaux d'existence, à tous les progrès de la vie; force qui dématérialise et libere la poussée ascensiomelle de l'esprit. Peur de lo chute, du néent, du cheos originel, de l'indifférencié. Blessure, déchirure pour atteindre le caché, l'anima, l'inconscient, la source, la nuit qui inspire.

L'ertiste doit en quelque sorte s'anéantir pour conquerir l'éternité. Il doit atteindre le noir de lo nuit, du rêve, du sommeil sacré où toute connaissance distincte est disparue et réduite au virtuel. La connaissance de la nuit fait revenir le créateur à lindéterminé, \& la purification de l'intellect oû l'inconscient se libère, où la germination riche de toutes les virtualités eclate en manifestation de vie. 


\section{CONCLUSION}

La tendance naturelle de toute vie est ascensionnelle. "Homme de Popier" dévolle la nécessaire obligation pour tous les "êtres pensants" de travailler de toute leur force pour atteindre individuellement et collectivement la cime, le sommet du cone. "Homme de Papier" témoigne de cette iuresse, de ce magnétisme des hauteurs qui nous etreint lorsquion frôle des crevasses, des abimes toujours présentes. "Homme de Fapier" essale de suivre l'Homme dans sa marche qui l'élève de son essence brute et élémentaire jusqu'à cette montée transcendante, le faite où il pourra trouver son accomplissement. Ce ne sera pas sens embûches, sans obstacles; le chemin sera parfois difficile et l'issue, victoire ou défaite, incertaine. Progression lucide ou dechéance dens lobscurite? "Homme de Papier", supra-conscient, métalengage du subsconscient surconscientisé. Papier matière, fidèle compagnon qui a permis à lhumanite sa plus grande évolution; médium apte à refléter le visage. la marche et le devenir de liHomme.

Préoccupation personnelle, sociale, philosophique, réalite la 
plus intime, dévollée. Vers quoi nous dirigeons-nous? Lavenir est-il prévisible? A-t-il un sens? LHumanite sunifie-t-elle dans le sens de lhistoire qui est liberstrice personnalisante, "amorisante" ou dans le sens de l'histoire qui nole lindividu dons la foule et le laisse perir de facon anarchique? "Homme de Papier" esseie d'y répondre dans un langege empreint despoir. Mú per une force qui transcende, il est arrivé à terme. Son vouloir-faire étant assez ferme pour ne reculer devant aucun enmui, aucun décoursgement, aucune peur en chemin. II sest ajuste, il a rectifie, stabilise sa marche avec une perception plus nette du but at ateindre. Il se devsit de créer une solidarité, une atmosphere cosmique. Cinq, quinze risquent moins qu'un seul de se tromper dans leur effort. Chaleur toujours plus claire, plus chaude à mesure qu'il progresse, qu'il entrevoit lissue. Rien ne saurait. apparemment empècher "Homme de Papier" de grandir en dépit de lo mort possible en avant ou qui rode autour de lui. Tout comme lhomme-individu devent le bien ou le mal, il gorde au coeur is possion de croitre et ne désespère jamais du mouvement qui l'sppelle en svant. Marche vers la "noosphère", recherche de la clef de voúte, de l'unite supérieure absolue. "Homme de Papier" he formant plus qu'une seule conscience. Etre qui domine le fremissement des êtres! océon tranquille où chaque goutte a conscience de demeurer elle-même! "Homme de Papier" complexe comme l'univers, un comme l'Homme; approche du mystère du monde où de lhumus indifférenciée il capte et transmue la substance pour la projeter dens l'infini de l'espace. 


\section{BIBLIOGRAPHIE}

BARTHES, Roland (1982) Lobvie et lootus, essals critiques $111 /$ Roland Berthes Paris, Seuil, $282 \mathrm{p}$

BARTHES, Rolond (1985) L'oventure sémiologique. Paris, Seuil, $358 \mathrm{p}$.

BAUDRILLARD, Jean (1976) L'échange symbolique et la mort, Paris, Gellimard, $347 p$.

CHAUCHARD, PaUl (1985) Tellhard de Chardin, Montrél, Ed. Du Levoin, $68 \mathrm{p}$.

FRANCE, CENTRE GEORGES POMPIDOU (1983) Le Papier, Revue Troverses, 27/28, Paris, $224 \mathrm{p}$.

HUNTER, Dard (1978) Papermaking, New York, Dover Publication., $611 \mathrm{p}$.

HUYGHE, René (1965) Lespuissances de limage: bilan d'une psychologie de l'art, Paris, Flammarion, $178 \mathrm{p}$.

JACQUARO, Albert (1991) Voici le temps du monde fini, Louisville, Ed. du Seuil, $183 \mathrm{p}$.

LACAN, Jacques (1966) Ecrits: par Jacques Lacon, Paris, Ed du Seuil, $924 p$.

LUND, Steffen Nordhal (1981) L'oventure du signifiant: une lecture de Barthes, Paris, Presses Universitaires de France, 124 .

MALRAUX, André (1965) Le musée imaginaire. Les voix du silence, (vol. 1), Paris, Gallimard, $149 \mathrm{p}$.

PASSERON, René (1986) L'oeuure picturale et les fonctions de lepparence, Paris, J. Vrin, $371 \mathrm{p}$. 
SIBoNy, Doniel (1972) Entre dire et faire, Paris, Bermard Grasset, 3970.

TEILHARD DE CHARDIN, PIerre (1955) Lephenomène humain, Paris, Ed. du Seuil, $347 \mathrm{p}$.

THIBAULT-LAULAN, Anne-Morie (1972) Image et communication, Paris, Ed. Universitaire, 1910.

TOUSSAINT, Bernard (1978) Qu'est-ce que lo semiologie? Toulouse, Privat, 179 p. 
ANNEXE 


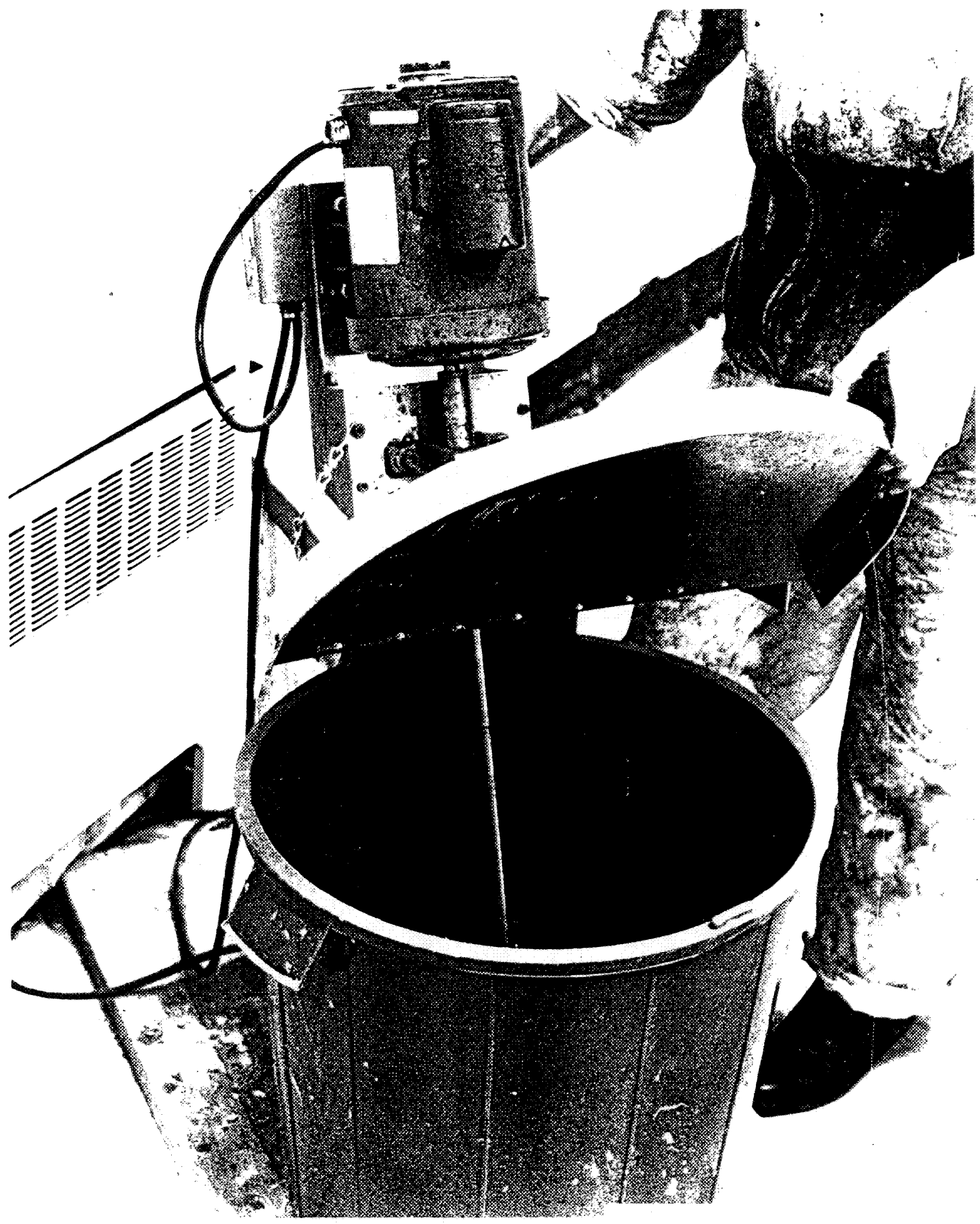

DEFIBRAGF DE WA PUPE D'ABACA 


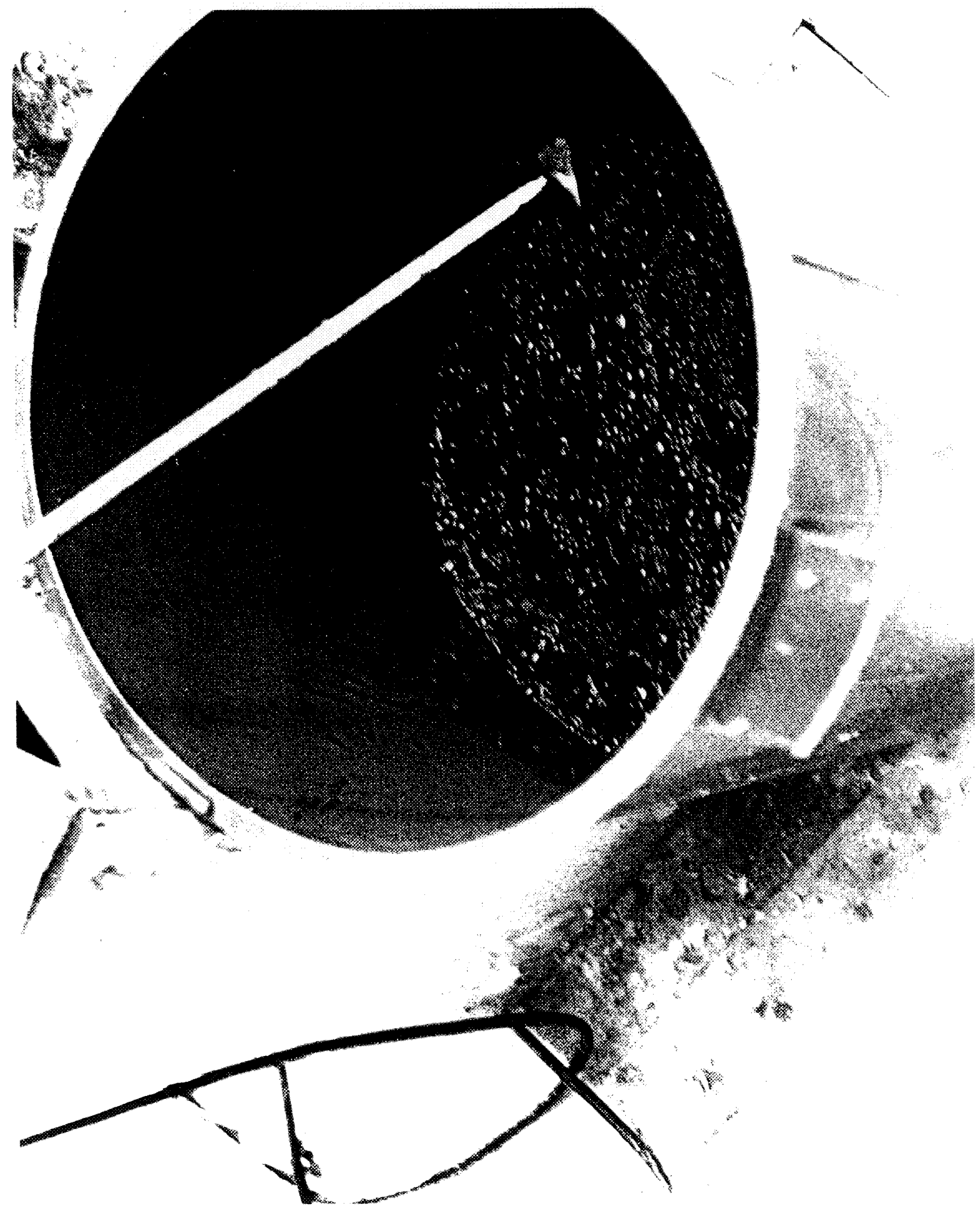

PATE DARACA 


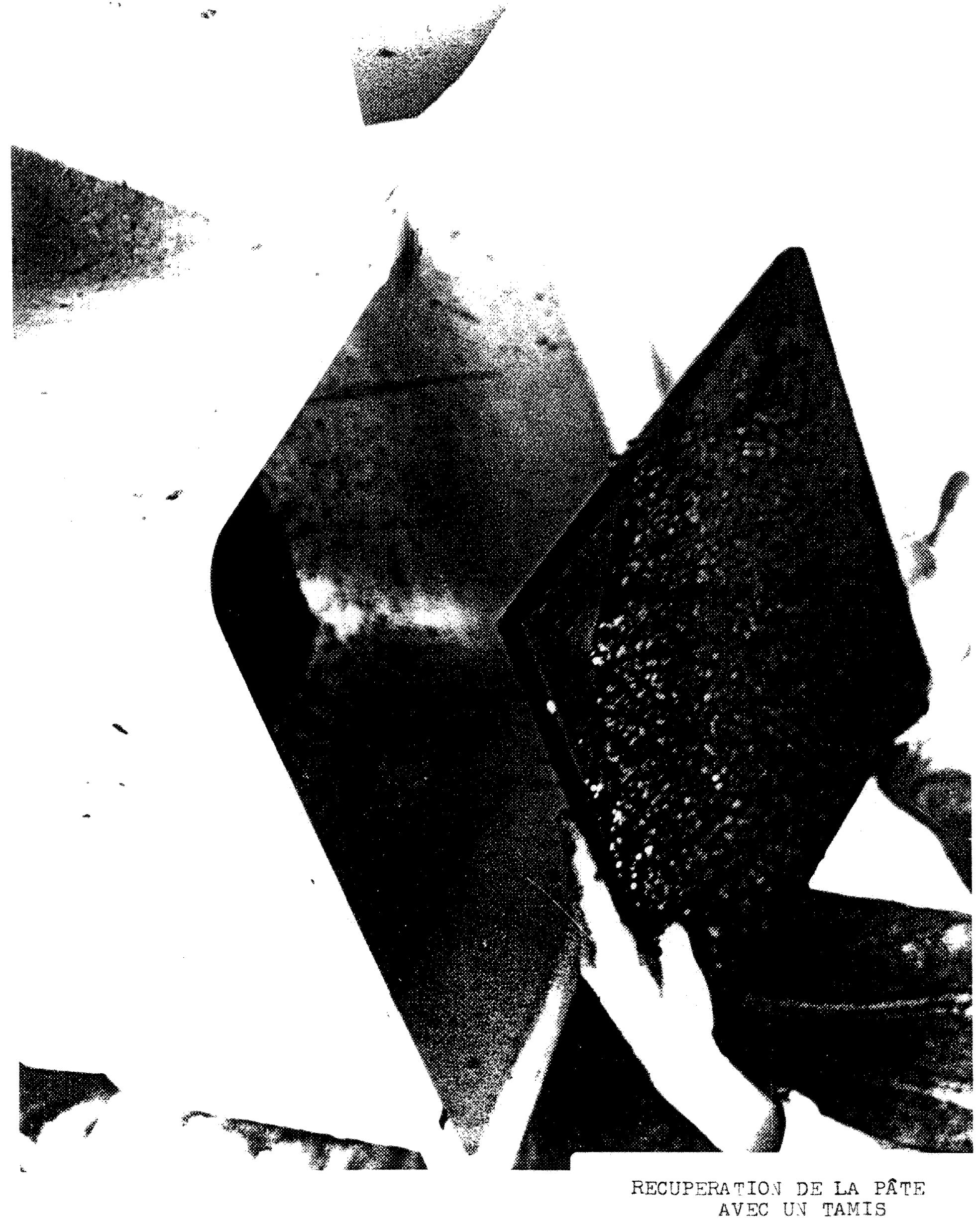




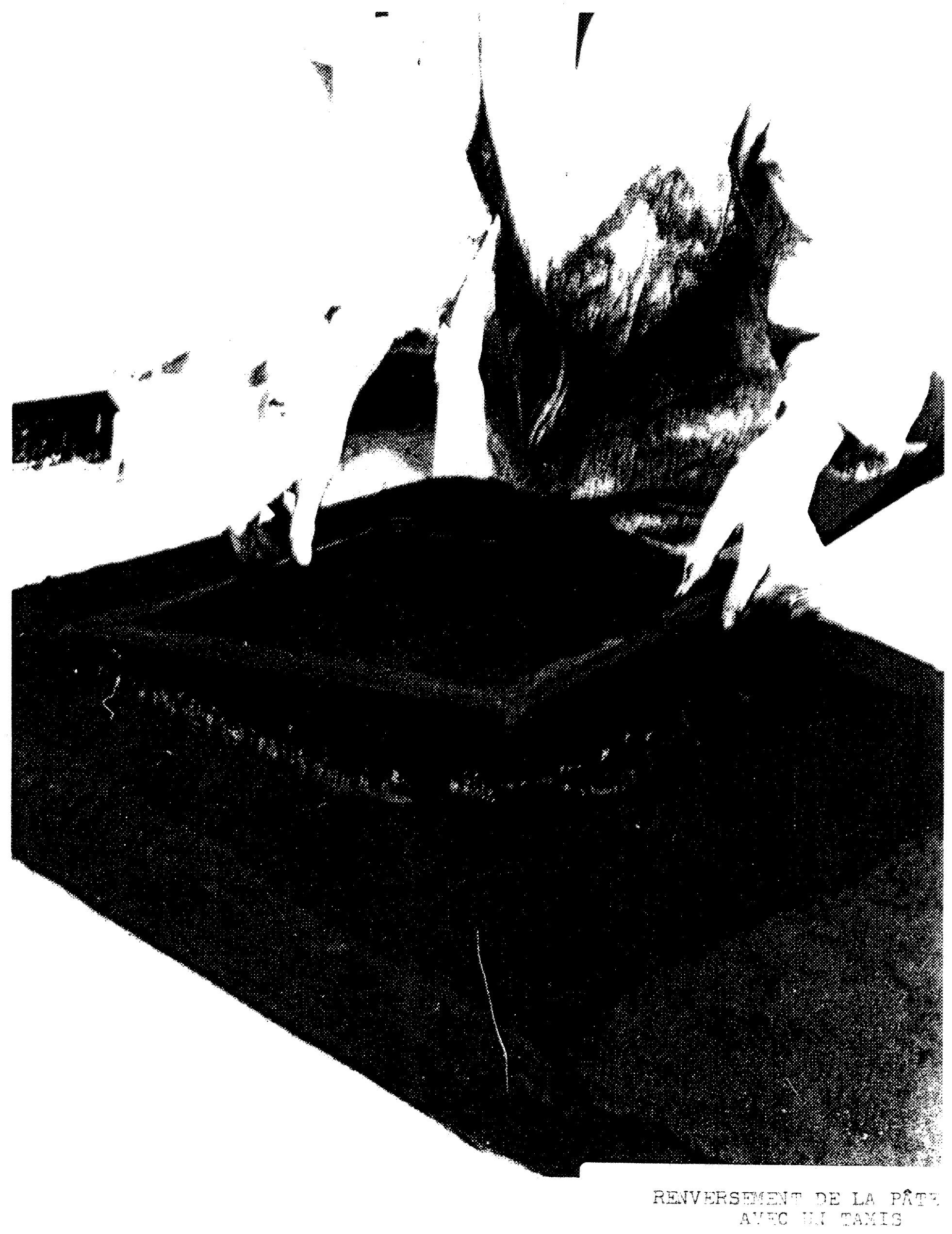




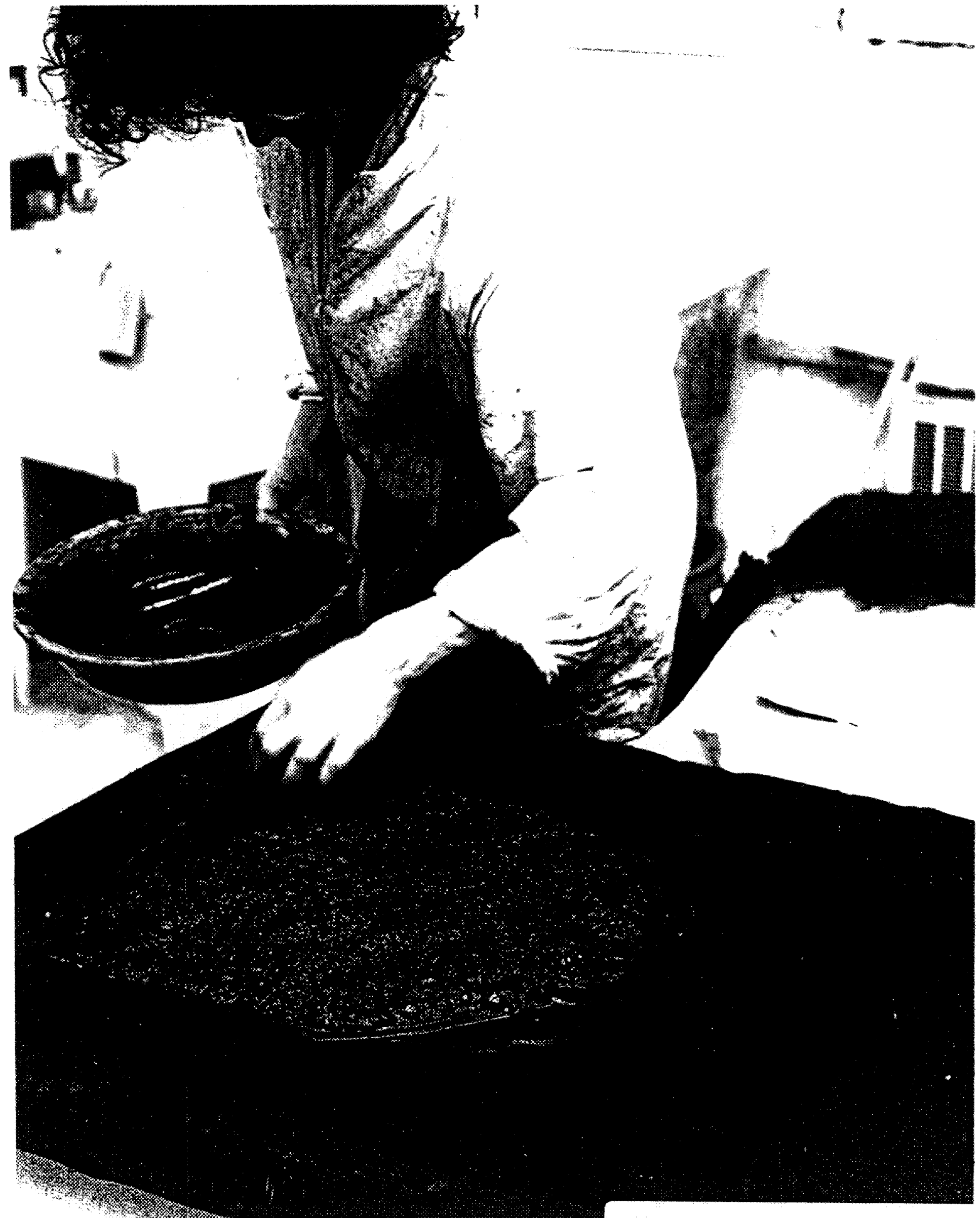





FFUILIS DE PAPTEF D'AMCA ADEA BOCUAG 







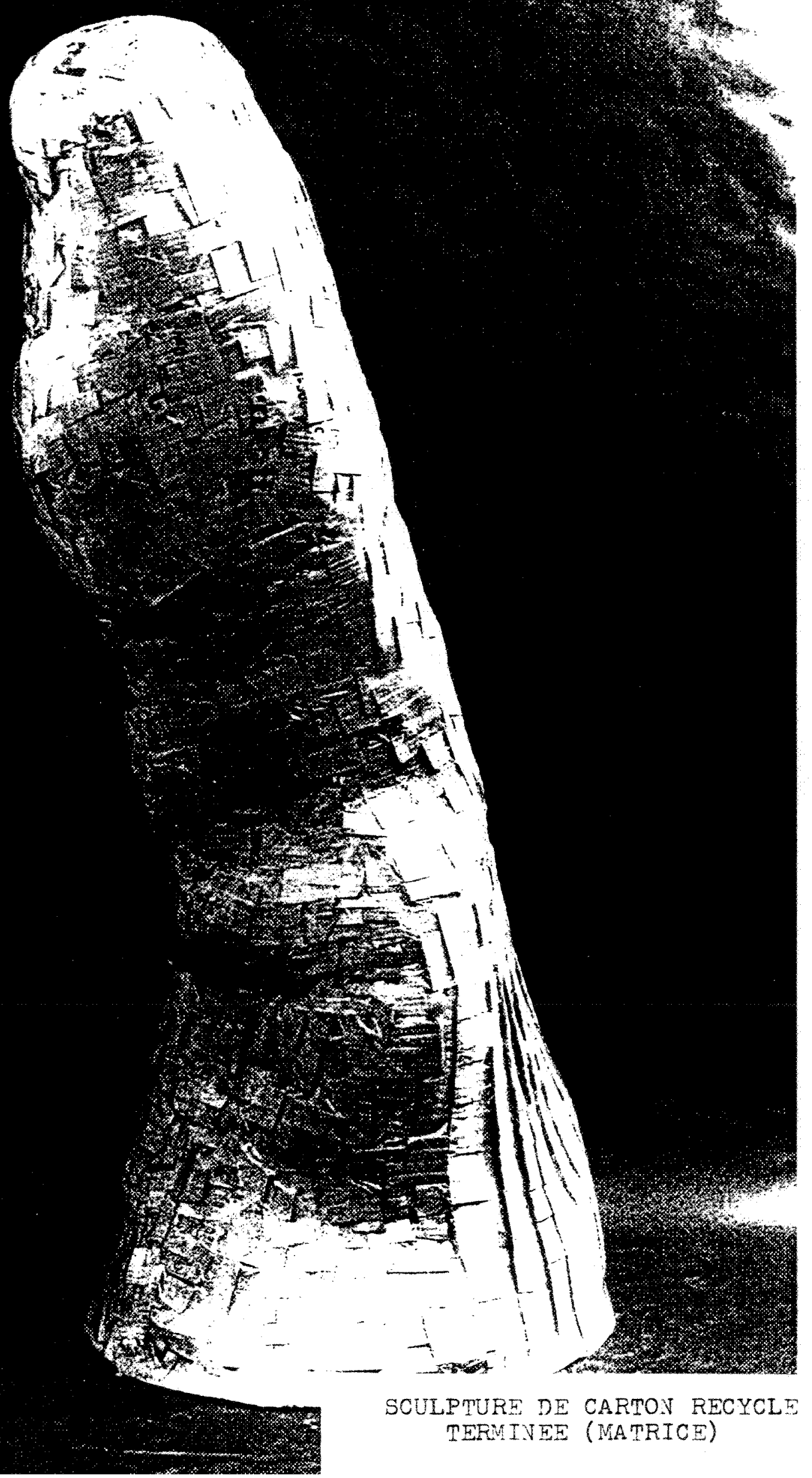

